Casos Clínicos

Arch. Esp. Urol. 2010; 63 (3): $231-234$

\section{VÁLVULAS URETERALES EN EL NIÑO. TRATAMIENTO MÍNIMAMENTE INVASIVO MEDIANTE LASER HOLMIUM}

\author{
José Antonio March Villalba, Agustin Serrano \\ Durbá, Carlos Dominguez Hinarejos, Francisco \\ Boronat Tormo', Francisco Estornell Moragues, Cinta \\ Sangüesa ${ }^{2}$ y Fernando García Ibarra.
}

Departamento de Urología Pediátrica, Unidad de Litotricia y Endourología' y Departamento de Radiología Pediátrica ${ }^{2}$. Hospital Universitario la Fe. Valencia. España.

Resumen.- OBJETIVOS: Presentar el manejo terapéutico mínimamente invasivo de la obstrucción unilateral del tracto urinario superior producida por una válvula ureteral.

MÉTODO: Descripción del caso clínico, la técnica endourológica utilizada y de los resultados a medio plazo, con apoyo de la literatura publicada al respecto.

RESULTADOS: Paciente de 12 años con clínica de dolor en flanco derecho. En la ecografía presentó ureterohidro- nefrosis derecha con parénquima conservado. La Uro-resonancia magnética reveló la presencia de un defecto de repleción en el tercio medio del uréter derecho y descartó una compresión extrínseca. En la ureterorrenoscopia se observó la válvula ureteral que fue resecada mediante laser holmium. La derivación ureteral se mantuvo 3 semanas. Tras 12 meses del tratamiento la paciente presentó ausencia de clínica y de ureterohidronefrosis derecha en ecografías de control.

CONCLUSIONES: El tratamiento endourológico mediante resección de la válvula ureteral obstructiva usando el laser Holmium Dormier es un método poco invasivo seguro y eficaz a corto-medio plazo.

Palabras clave: Válvula ureteral. Endourología. Ureterohidonefrosis. Láser Holmium Dornier.

Summary.- OBJECTIVES: To present the minimally invasive management of an annular ureteric valve causing upper urinary tract obstruction.

METHODS: A case report description: endourological technique and medium-term results with review of the published reports.

RESULTS: A 12 year old patient with right flank pain was attended. Ultrasound showed right ureterohydronephrosis with conserved parenchyma. Uro-MRI (axial plane) showed dilation down to the middle of the right ureter with a filling defect, without extrinsic compression. Ureterorenoscopy showed a ureteral valve that was resected by holmium laser. Ureteral stent was maintained for 3 weeks. Twelve months after treatment patient has no symptoms and the following ultrasounds did not reveal right ureterohydronephrosis.

CONCLUSIONS: Obstructive ureteral valve resection by endourological treatment using a Dornier holmium laser is a safe and effective minimally invasive method according to short and medium term results.

Keywords: Ureteric valve. Endourology. Ureterohidronephrosis. Laser Holmium Dornier.

\section{INTRODUCCIÓN}

Las válvulas ureterales congénitas son una entidad clínica poco frecuente, pueden estar asociadas a otras anomalías congénitas urológicas (1).

Su origen es intraútero debido a una recanalización inadecuada del uréter (persistencia de la membrana epitelial de Chwalle más allá de las 6 semanas de gestación) - a un aporte vascular insuficiente (2). Están formadas histológicamente como pliegues transversos de la muco- 
sa ureteral que contienen paquetes de fibras de músculo liso (1).

La ureterorrenoscopia en el niño ha sido aplicada de manera efectiva en el tratamiento con laser holmium de diversas patologías como, por ejemplo, la obstrucción de la unión pieloureteral o la estenosis de la unión ureterovesical (3).

Describimos un caso de una paciente en edad pediátrica que presenta una válvula en el uréter derecho en situación medial, tratada mediante el uso del laser holmium Dornier.

\section{PRESENTACIÓN}

Niña de 12 años que refería dolor en flanco derecho de un mes de evolución. La ecografía del sistema excretor reveló una ureterohidronefrosis derecha (diámetro anteroposterior de la pelvis renal de $52 \mathrm{~mm}$ ) con una correcta diferenciación córtico-medular (espesor de 4,4 $\mathrm{mm})$. La creatinina sérica se encontró dentro de valores normales $(0,89 \mathrm{mg} / \mathrm{dl})$. El renograma diurético demos- tró la existencia de un patrón obstructivo (alargamiento de la fase excretora de la curva del sistema derecho con respecto al contralateral). La función renal diferencial fue de $47 \%$ y $53 \%$ en el riñón derecho e izquierdo respectivamente.

La tomografía computerizada (TC) no identificó ninguna etiología extraluminal ni un defecto en el uréter distal derecho. La uro-resonancia magnética (Uro-RM) (Gd Multihance) tampoco evidenció una causa extrínseca, confirmando la dilatación por encima del defecto de repleción (Figura 1).

\section{MATERIAL Y MÉTODOS}

El procedimiento técnico realizado para tratar dicha obstrucción consistió en un abordaje endourológico (ureterorrenoscopio 7,5 ch) para poder utilizar el laser holmium Dornier como fuente de energía de resección de la válvula ureteral. La cistoscopia mostró la existencia de 2 meatos ureterales ortotópicos. Mediante la ureterorrenoscopia pudimos observar como la luz ureteral se estrechaba en la zona de la válvula ureteral (dia-
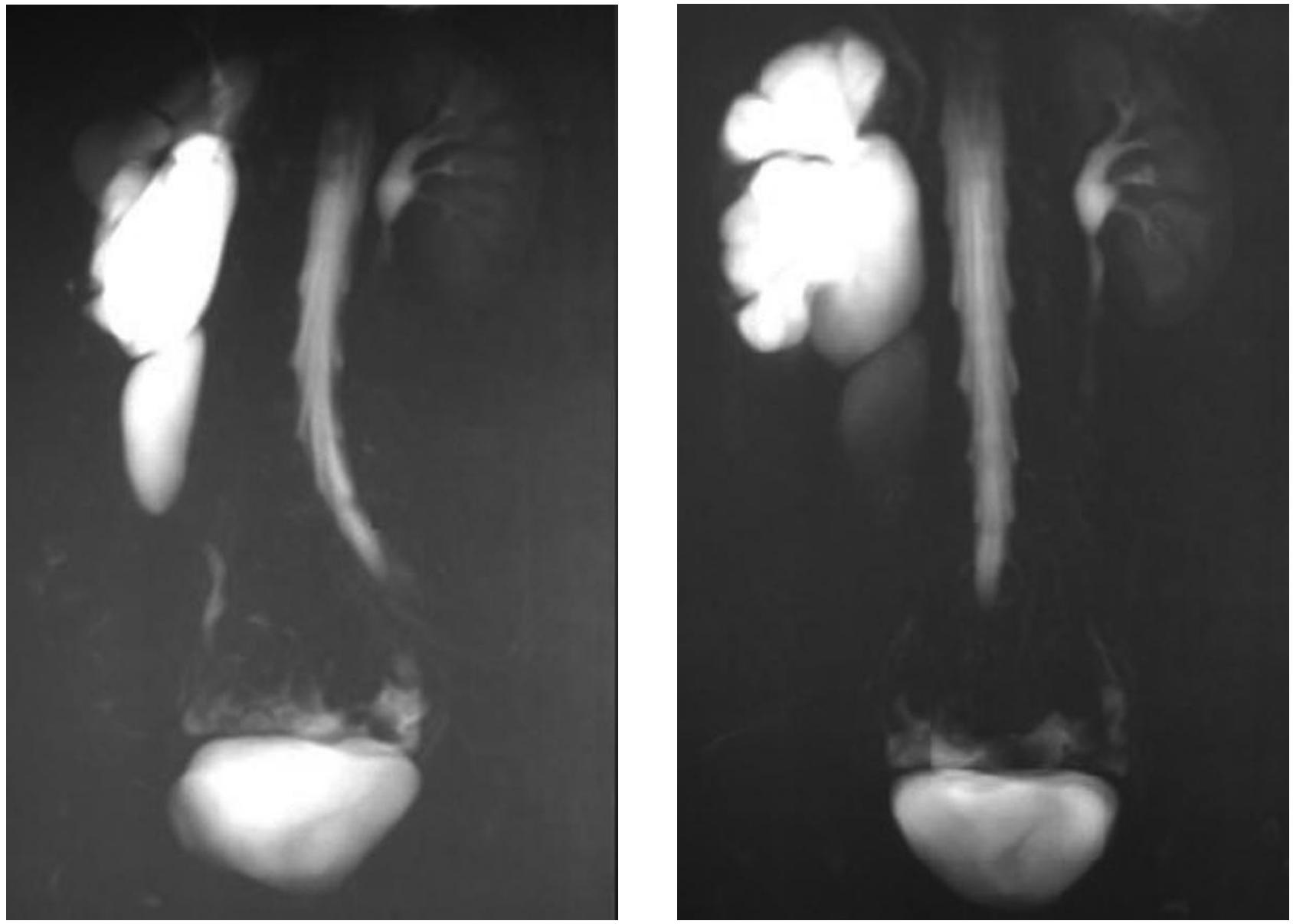

FIGURA 1. Uro-RM mostrando una estenosis en la porción medial del uréter medio causante de ureterohidronefrosis. 
fragmática y estenótica) a nivel de L3-L4. La pielografía retrógrada practicada evidenció el defecto de relleno en el tercio superior del uréter derecho y el paso de contraste a pelvis y cálices tras apertura de la estenosis (Figura 2).

Se realizó la ablación en círculo (iniciándola en la zona posterolateral) del tejido valvular mediante el uso de fibras laser de $200 \mu$ de diámetro con una potencia de energía de 4 Vatios $(0,2-0,8 \mathrm{~J}$ a $3-10 \mathrm{~Hz})(0,5 \mathrm{~J} \times 8 \mathrm{~Hz})$. Tras la resección se colocó un catéter doble jota de $7 \mathrm{Ch}$ que se mantuvo 3 semanas.

Tras 12 meses de seguimiento se observó una notable mejoría clínica, remitiendo la dilatación pieloureteral derecha en ecografías de control, sin producirse recurrencia del proceso.

\section{DISCUSIÓN}

Las válvulas ureterales congénitas son poco frecuentes. Existen publicados sólo 60 casos (3 corresponden a la

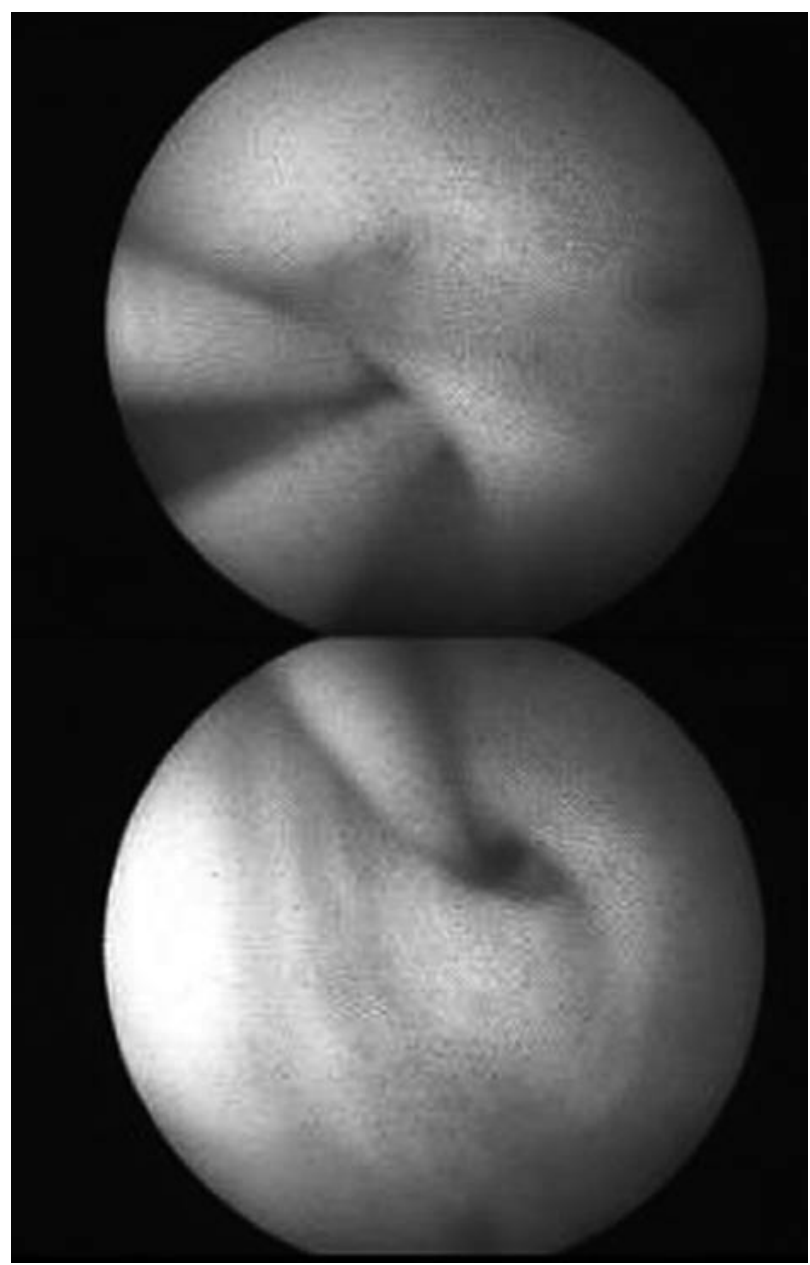

literatura científica española) desde que Wolfler las describiera en 1877 (1-4).

Suelen estar asociadas a otras anomalías congénitas del aparato urinario como el reflujo vesicoureteral (la más frecuente), duplicación ureteral, uréter ectópico, riñón hipoplásico contralateral o agenesia renal $(1,5)$. Pueden presentar una obstrucción que genere hidronefrosis y dolor tipo cólico o sordo en flancos.

Cabe realizar el diagnóstico diferencial con el megauréter, procesos obstructivos extrínsecos y obstructivos intrínsecos como la estenosis de la unión pieloureteral o de la unión ureterovesical. Para el diagnóstico de válvulas ureterales se tienen que cumplir:

a) Presencia en el examen histológico de un pliegue transverso de la mucosa ureteral que contenga fibras de músculo liso,

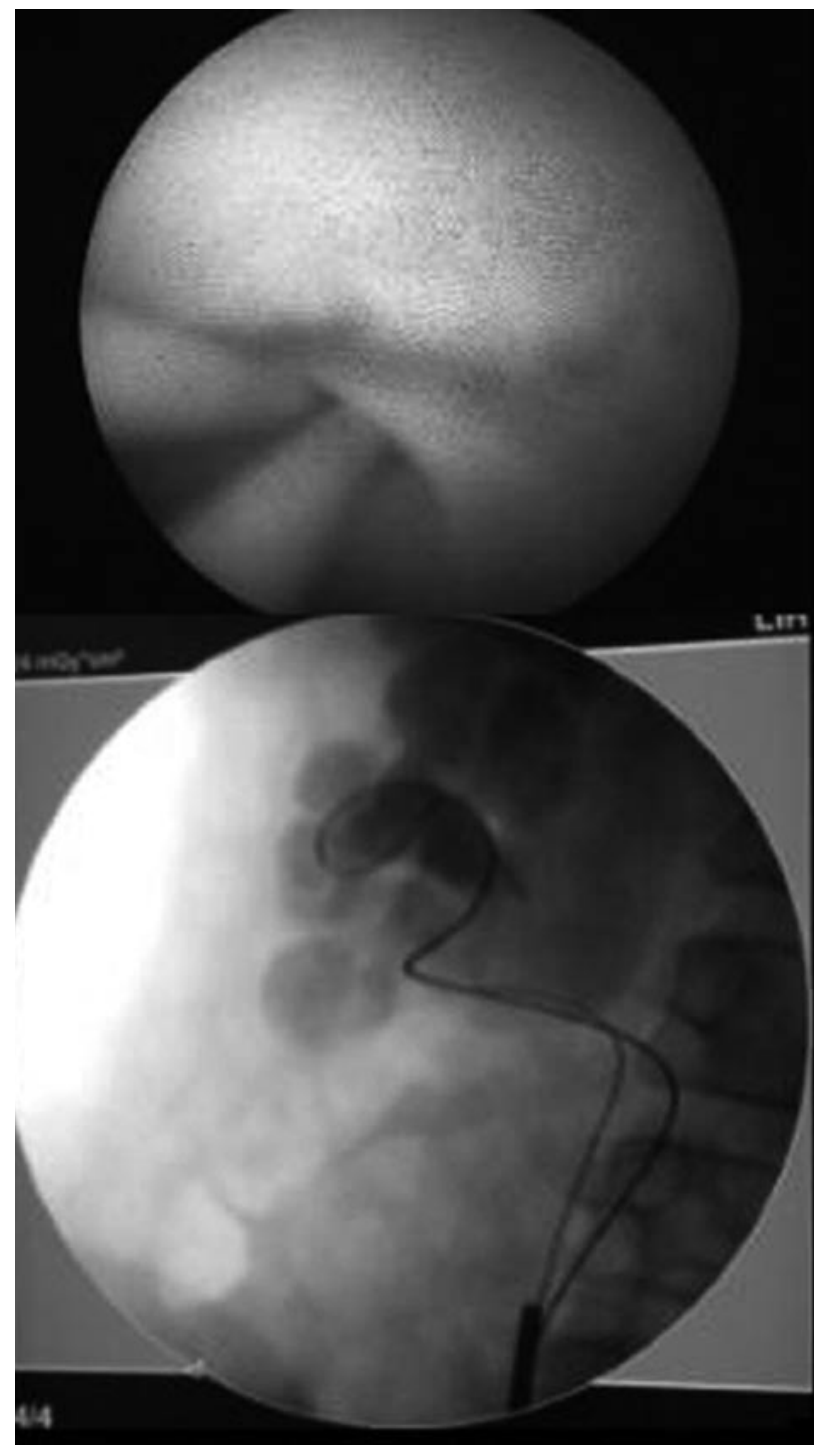

FIGURA 2. Abordaje endourológico de la válvula ureteral donde se muestra la estenosis y la ureterohidronefrosis causada y el pielograma retrógrado durante la resección. 
b) signos de enfermedad obstructiva por encima de la válvula y de un uréter normal por debajo de ésta y

c) ninguna evidencia de otra etiología que produzca una obstrucción mecánica o funcional (1).

La ecografía en una herramienta diagnóstica muy útil de inicio ya que confirma la presencia de dilatación de la vía excretora, pero la Uro-RM ofrece un diagnóstico morfológico en diversos planos de la etiología de la obstrucción, pudiendo discriminar si existe una causa extra o intraluminal. Aunque en este caso hemos utilizado también la TC, consideramos que la Uro-RM es una técnica diagnóstica de imagen útil en estos casos ya que evita la exposición del niño a radiación hecho que no ocurre con la TC o la urografía intravenosa, aunque la limitación que presenta es la necesidad de sedación del paciente.

El renograma diurético es también de gran utilidad en el manejo diagnóstico ya que informa de de la funcionalidad renal y del grado y nivel de obstrucción.

Aunque el tratamiento convencional de las válvulas ureterales ha sido la escisión quirúrgica del segmento ureteral que contiene la válvula seguido de una anastomosis ureteral término-terminal o de un reimplante si ésta se localiza en el tercio distal del uréter $(1,6)$, este tipo de obstrucción puede ser tratado mediante un abordaje endourológico, atendiendo al desarrollo tecnológico y el perfeccionamiento de la endourología del adulto que permite una efectiva aplicación a la patología pediátrica $(3,5)$.

Es aconsejable realizar una pielografía ascendente en el momento del tratamiento endourológico ya que permite una visualización mediante fluoroscopia del uréter por encima y por debajo de la obstrucción, y así controlar mejor la resección de la válvula ya que nos permite observar un aumento del flujo retrógrado a tercio superior del uréter y a pelvis del contraste tras la eliminación de la estenosis así como del extravasado de éste si se produce una rotura ureteral $(5,6)$.

La resección endoscópica de la válvula ha sido descrito mediante abordajes tanto anterógrados (mediante nefroscopia con corte frío, cortando la cara dorsal de la válvula) como retrógrados mediante el uso de un ureterorrenoscopio que permita poder introducir las fibras del laser Holmium $(2,8)$. El abordaje retrógrado en niños con estenosis ureterales es seguro y efectivo como también se demuestra en nuestro caso (3).

Al optar por la resección de la válvula carecemos de material histológico que pueda describir la presencia de fibras de músculo liso, pero pensamos que éste sería sólo un diagnóstico de confirmación.

Aunque la evolución de la paciente es satisfactoria, no podemos descartar que aparezca una estenosis por fi- brosis de la zona resecada ya que el seguimiento es sólo de 12 meses.

\section{CONCLUSIONES}

La presencia de válvulas ureterales congénitas está dentro del diagnóstico diferencial de una hidronefrosis, aunque sea una entidad clínica poco frecuente. El tratamiento endourológico de la válvula ureteral con laser holmium es un tratamiento seguro y eficaz a corto plazo.

\section{BIBLIOGRAFÍA Y LECTURAS RECOMENDADAS ( ${ }^{*}$ lectura de interés $y^{* *}$ lectura fundamental)}

**1. Gupta RK, Borwankar SS, Parelkar SV. Ureteric valve: case report with an insight into anatomy, embryology, presentation and management. Indian J Urol 2008; 24: 561-3.

**2. Singh SK, Wadhwa P. Ablation of diafragmatic annular valve with holmium laser. Int Urol and Nephrol 2006; 38: 157-9.

**3. Sofer M, Binyamini J, Ekstein PM, Bar-Yosef Y, Chen J, Matzkin H, Ben-Chalm J. holmium laser ureteroscopic treatment of various pathologic features in pediatrics. Urology. 2007;69(3):566-9.

*4. Ricodesanz JM, Velasco E. Consideraciones en un caso de válvula ureteral congénita. Arch Esp Urol. 1964; 17: 238-45.

*5. Tomás M, Pérez Albacete M, Salmerón J, Falcó E, Rodríguez-Bermejo M. Válvula ureteral: presentación de un caso. Arch Esp Urol. 1984; 37: 541-4.

6. Álvarez F, Virseda JA, Bolaño N, Recio R, Melchor A, Orradre JL. Válvula ureteral: un nuevo caso. Actas Urol Esp. 1987; 11: 624-6.

7. Rossi E, Rodó Salas J, Cáceres Aucatoma F, Olivares Muñoz M, Morales Fochs L. Congenital ureteral valves: two new cases and review of the literature. J Pediatr Urol 2007; 3: 344-9.

*8. Nishio S, Hamada H, Yokoyama M. Endoscopic incision for the treatment of a ureteric valve. BJU Int 1999; 83: 1081-2.

**9. Di Mario M, Fruhwirth R, Barbuti D, Fasanelli S. Congenital valves of the ureter. Comments on $7 \mathrm{ca}-$ ses studied in childhood. Radiol Med 1992; 83: 8590 . 